(RESEARCH ARTICLE)

\title{
Modulation of synergism COX-1 with COX-2 in the tail flick of mice
}

\author{
Noriega Viviana 1, 2, Sierralta Fernando ${ }^{3}$, Sotomayor-Zárate Ramón 4, Prieto Juan Carlos 2,3 and Miranda \\ Hugo F 5, * \\ ${ }^{1}$ Faculty of Medicine, Clínica Alemana, Universidad del Desarrollo, Chile. \\ ${ }^{2}$ Cardiovascular Department; Clinical Hospital, Universidad de Chile, Santiago, Chile. \\ ${ }^{3}$ Pharmacology Program, ICBM, Faculty of Medicine, Universidad de Chile, Santiago, Chile. \\ ${ }^{4}$ Laboratory of Neurochemistry and Neuropharmacology, Faculty of Sciences Universidad de Valparaíso, Valparaíso, \\ Chile \\ ${ }^{5}$ Neuroscience Department, Faculty of Medicine, Universidad de Chile, Santiago, Chile.
}

Publication history: Received on 11 June 2020; revised on 20 June 2020; accepted on 23 June 2020

Article DOI: https://doi.org/10.30574/wjarr.2020.6.3.0200

\begin{abstract}
Nonsteroidal anti-inflammatory drugs (NSAIDs) are often used in the treatment of pain. In this study was evaluated, by isobolographic analysis, in a radiant heat model of mice, the tail flick, the potential antinociceptive pharmacological interaction of the combination between ketorolac and meloxicam and the modulation by tropisetron, risperidone, prazosin, yohimbine, naltrindole or 7-Nitroindazole. In the assay, the combination of ketorolac with meloxicam induces an additive interaction with an interaction index of $12.20 \pm 0.92$. Neither tropisetron nor risperidone nor prazosin nor yohimbine modify the nature of interaction, however naltrindole and 7-Nitroindazol change the nature of interaction from additive a subadditive with an interaction index of $16.85 \pm 2.52$ and $18.00 \pm 2.57$, respectively. The current results suggests that the interaction antinociceptive of the combination between ketorolac with meloxicam does not seem to be influenced by adrenergic, dopaminergic, histaminergic or serotonegic mechanisms. Nonetheless, the pretreatment of the mice with naltrindole or con 7-Nitroindazole induced a significant decrease in the antinociceptive power of the combination with a significant reduction in DE. It is suggested that DOR and NO may be involved, in the tail flick model, regulating the antinociceptive bioactivity of these NSAIDs.
\end{abstract}

Keywords: NSAIDs; Tail flick; Isobolographic analysis; Receptors antagonist

\section{Introduction}

Pain is a consequence caused by tissue damage and consecutively produces the activation of receptors in the primary afferent fibers. Pain has been mainly classified as nociceptive, inflammatory and neuropathic.

Nociceptive pain (nociception) corresponds to the response to a harmful stimulus in which the nociceptors of the endings of muscles, skin, bones and different organs are activated. Nociceptors are capable of detecting a variety of stimuli, whether thermal, chemical, or mechanical. Inflammatory pain is described as the tissue response to damaging stimuli to eradicate necrotic cells and initiate tissue repair. It is usually divided by reason of time into chronic and acute pain. Among the mediators described in the lesion, histamine, serotonin, kinins, nitric oxide, glutamate and others should be mentioned [1]. Neuropathic pain is described as injury or nerve damage, generated by various metabolic diseases or inflammation. In this pain, the involved nociceptors present a high interaction, with ion channels, such as those of voltage-dependent NA, aimed at neuronal excitability and propagation of action potentials.

The pharmacotherapeutic treatment of the various types of pain principally uses NSAIDs, mostly analgesic, antipyretic and anti-inflammatory agents. The mechanism of action of these drugs is to inhibit the three cyclooxygenases (COX-1,

\footnotetext{
${ }^{*}$ Corresponding author: Miranda Hugo F
} 
COX-2 and COX-3), and thus interrupt the development of biosynthesis of prostanoids involved in pain. COX-1 is constitutively expressed in various tissues such as blood vessels, kidneys, gastrointestinal tract. COX-2 is inducible in inflamed tissues, and kidneys. COX-3 is expressed in nerve tissues, such as the spinal cord, cerebral cortex, and end othelial cells and the heart. The various NSAIDs differ in their selectivity of action for each COX, with COX-1 inhibitors being primarily antinociceptive and COX-2 inhibitors anti-inflammatory $[2,3]$.

Experimental studies with animals have been planned that allow specific characteristics of the stimulus and the behavior of the animal, there are different tests and the most used are the short duration of the stimulus or inducer of phasic pain, among them the movement of the tail, as the tail flick. In phasic pain tests, it is common to evaluate the antinociceptive effect of drugs, either alone or in combination, using so-called multimodal analgesia. This type of analgesia occurs due to interactions between drugs, mainly of pharmacodynamic nature, in which the effect of one is altered by the action of the other. The interactions can be classified as synergistic, additive or antagonistic in nature, with isobolographic analysis in place that allows them to be classified safely and effectively unequivocally. The synergistic interactions are those that have a greater application, since they allow to improve the analgesic potential, decrease the doses and, at the same time, significantly reduce the adverse reactions of the components of the combination $\{4,5\}$.

The objective of the present study was to evaluate by isobolographic analysis, the interaction of the NSAIDs combination (ketorolac and meloxicam) in a radiant heat model, the tail flick test and their possible modulation by tropisetron, risperidone, prazosin, yohimbine, naltrindole or 7-Nitroindazole.

\section{Material and methods}

\subsection{Animals}

CF-1 male mice, 28-30 g, housed in a $12 \mathrm{~h} \mathrm{light/dark}$ at $22^{\circ} \pm 1^{\circ} \mathrm{C}$, with free access to food and water and acclimatized to laboratory condition for at least 2 hours, were used. Experimental procedures were carried out in agreement with the Institutional Animal Care and Use Committee at the Faculty of Medicine, Universidad de Chile (Protocol CBA 0852/FMUCH/2018). Each animal was used only once and injected only one dose of the drugs tested and the behavior assay was performed by investigators blinded to the treatment. Animals were euthanized by overdose of anesthetic immediately after the algesiometer test. Control saline animals, two mice by group, were run interspersed concurrently with the drug treated mice, which prevented run as a simple group.

\subsection{Analgesia}

The evaluation of the analgesia was carried out by the tail flick assay, as previously described [6]. A focus of radiant heat (tail flick algesiometer, Ugo Basile, Comerio, Italy). The latency response was adjusted between 2 to 3 sec. A cut-off time of $8 \mathrm{sec}$ was imposed to avoid damage to the tail. The latencies responses were converted to \% maximum possible effect ( $\%$ MPE) according to:

$\% \mathrm{MPE}=\left[\left(\mathrm{T}_{1}-\mathrm{T}_{0}\right) /\left(\mathrm{T}_{2}-\mathrm{T}_{0}\right)\right] \times 100$,

where $\mathrm{T}_{0}$ and $\mathrm{T}_{1}$ are latencies control and after treatment, respectively and $\mathrm{T}_{0}$ is the cut-off time. The control latency was $2.54 \pm 0.11(n=24)$.

\subsection{Analysis isobolographic}

Isobolographic analysis was used to characterize interaction between ketorolac and meloxicam, according to previous described [7]. The isobologram is a graphical representation of isoeffective doses of ketorolac and meloxicam combined in fixed ratios (1:1) of the corresponding $E D_{25}$ previously determined separately for each drug. The isobologram is constructed by connecting the $\mathrm{ED}_{25}$ of ketorolac on the abscissa with the $\mathrm{ED}_{25}$ of meloxicam on the ordinate, fixing the line of additivity and the point of combination theoretical $E D_{25}$. Then, the point representing the experimental ED $25 \mathrm{Will}$ be located in the isobologram, and the site of the graph where is located determines the type of interaction. If the experimental point is below the line of additivity the effect of the combination is synergistic or superadditive. This means, that the effect obtained from the drug combination is greater and statistically different from that calculated theoretically with the same proportions. Furthermore, a measure of potency of the combination, the interaction index (I.I.), calculated (experimental $\mathrm{ED}_{25}$ / theoretical $\mathrm{ED}_{25}$ ) if the ratio is close to 1 the interaction is additive. Values lower than 1 the magnitude the interaction is synergistic and values higher than 1 correspond to sub-additive. 


\subsection{Protocol}

Dose-response curves i.p. for the antinociceptive effect of ketorolac (1, 3,10, and $30 \mathrm{mg} / \mathrm{kg}$ ) or meloxicam ( $3,10,30$ and $100 \mathrm{mg} / \mathrm{kg}$ ) were obtained using at least six to eight animals for each doses. From the log dose-response curves permitted the calculation of the dose that produced 25\% de antinociception (ED25) for each drug. The i.p. effect of tropisetron $(0.5 \mathrm{mg} / \mathrm{kg}$ ) or risperidone $(0.01 \mathrm{mg} / \mathrm{kg})$ or prazosin $(1 \mathrm{mg} / \mathrm{kg})$ or yohimbine $(1 \mathrm{mg} / \mathrm{kg})$ or naltrindole ( $1 \mathrm{mg} / \mathrm{kg}$ ) or 7-Nitroindazole ( $5 \mathrm{mg} / \mathrm{kg}$ ) was evaluated for the changes induced in the ED25 of the NSAIDs. The doses used were taken from the literature $[7,8]$

\subsection{Drugs}

All drugs freshly dissolved in a sterile physiological saline solution on a constant volume of $10 \mathrm{ml} / \mathrm{kg}$ and administered intraperitoneally (i.p.). Saval Laboratories Chile provided meloxicam, ketorolac trometamol from Laboratory Chile, risperidone from Royal Pharm SA, prazosin hydrochloride, yohimbine hydrochloride, naltrindole hydrochloride and 7Nitroindazol were purchased from Sigma-Aldrich Chemical Co, St. Louis, MO, USA. USA

\subsection{Statistical analysis}

All results are expressed as means \pm standard error of mean (SEM) or $\mathrm{ED}_{25}$ value with $95 \%$ CL and were analyzed using a one-way ANOVA followed by Tukey's post hoc test. All calculations were performed with the program Pharm Tools Pro, version 1.27, Mc Cary Group Inc., PA, USA. P values under $0.05(\mathrm{p}<0.05)$ were considered significant.

\section{Results}

\subsection{Ketorolac and meloxicam antinociception in the mice tail flick}

The i.p. administration of ketorolac or meloxicam produced a dose-dependent antinociceptive effect in the tail flick of mice with an $\mathrm{ED}_{25}$ of $8.56 \pm 0.91 \mathrm{mg} / \mathrm{kg}$ for ketorolac and $18.15 \pm 1.55 \mathrm{mg} / \mathrm{kg}$ for meloxicam. The relative analgesic potency, based on the equieffective doses, resulted to be ketorolac 2.12 times more potent than meloxicam. All these data are shown in Table 1.

Table 1 ED25 values with SEM in $\mathrm{mg} / \mathrm{kg}$ and analgesic ratio (AR) of the antinociception of ketorolac and meloxicam in the tail flick of mice

\begin{tabular}{lcc}
\hline Drug & ED25 \pm SEM $(\mathbf{m g} / \mathbf{~ k g})$ & AR \\
\hline Ketorolac & $8.56 \pm 0.91$ & 2.12 \\
Meloxicam & $18.15 \pm 1.55$ & 1.00 \\
\hline
\end{tabular}

\subsection{Interaction between ketorolac with meloxicam}

The co-administration i.p. of ketorolac with meloxicam was additive with an interaction index of 1.065 . The experimental ED25 was $12.20 \pm 0.92 \mathrm{mg} / \mathrm{kg}$, as can see in Table 2 .

\subsection{Effect of naltrindole and 7- Nitroindazole on ketorolac plus meloxicam isobologram}

Pretreatment of mice with $1 \mathrm{mg}$ / kg i.p. of naltrindole (NTI) or 5 mg / kg i.p. of 7-Nitroindazole, doses with no effect on the basal behavior of the mice, resulted in a subadditive interaction, with a significant increase in DE25 and an interaction index of 1,380 and 1,475, respectively. Furthermore, DE25 increased significantly to $16.85 \pm 2.52$ and 18.00 \pm 2.57 , respectively. All data are shown in Table 2 and Figure 1 . 
Table 2 Experimental ED25 \pm SEM in $\mathrm{mg} / \mathrm{kg}$ and interaction index (I.I.) values for the antinociceptive activity of synergic combination of ketorolac with meloxicam before and after antagonists pretreatment in the tail flick of mice.

\begin{tabular}{llll}
\hline & Experimental & I.I. & Interaction \\
\hline KETO/MELO & $12.20 \pm 0.92$ & 1.065 & Additive \\
+ Tropisetron & $11.80 \pm 1.80$ & 0.967 & Additive \\
+ Risperidone & $12.40 \pm 1.96$ & 1.016 & Additive \\
+ Prazosin & $13.80 \pm 2.75$ & 1.130 & Additive \\
+ Yohimbine & $14.00 \pm 2.29$ & 1.163 & Additive \\
+ Naltrindole & $16.85 \pm 2.52$ & 1.380 & Subadditive \\
+ 7-Nitroindazol & $18.00 \pm 2.57$ & 1.475 & Subadditive \\
\hline
\end{tabular}

\subsection{Effect of tropisetron, risperidone, prazosin and yohimbine on ketorolac plus meloxicam isobologram}

Pretreatment of mice with $0.5 \mathrm{mg} / \mathrm{kg}$ i.p. of tropisetron (TROPI) or $0.01 \mathrm{mg} / \mathrm{kg}$ i.p. risperidone (RISPER) or $1 \mathrm{mg} / \mathrm{kg}$ i.p. prazosin (PRAZO) or $1 \mathrm{mg} / \mathrm{kg}$ i.p. of yohimbine (YOHIM), doses of the drugs that had no effect on basal nociception and that did not induce significant modifications of the previous additive interaction of the combination of ketorolac with meloxicam.
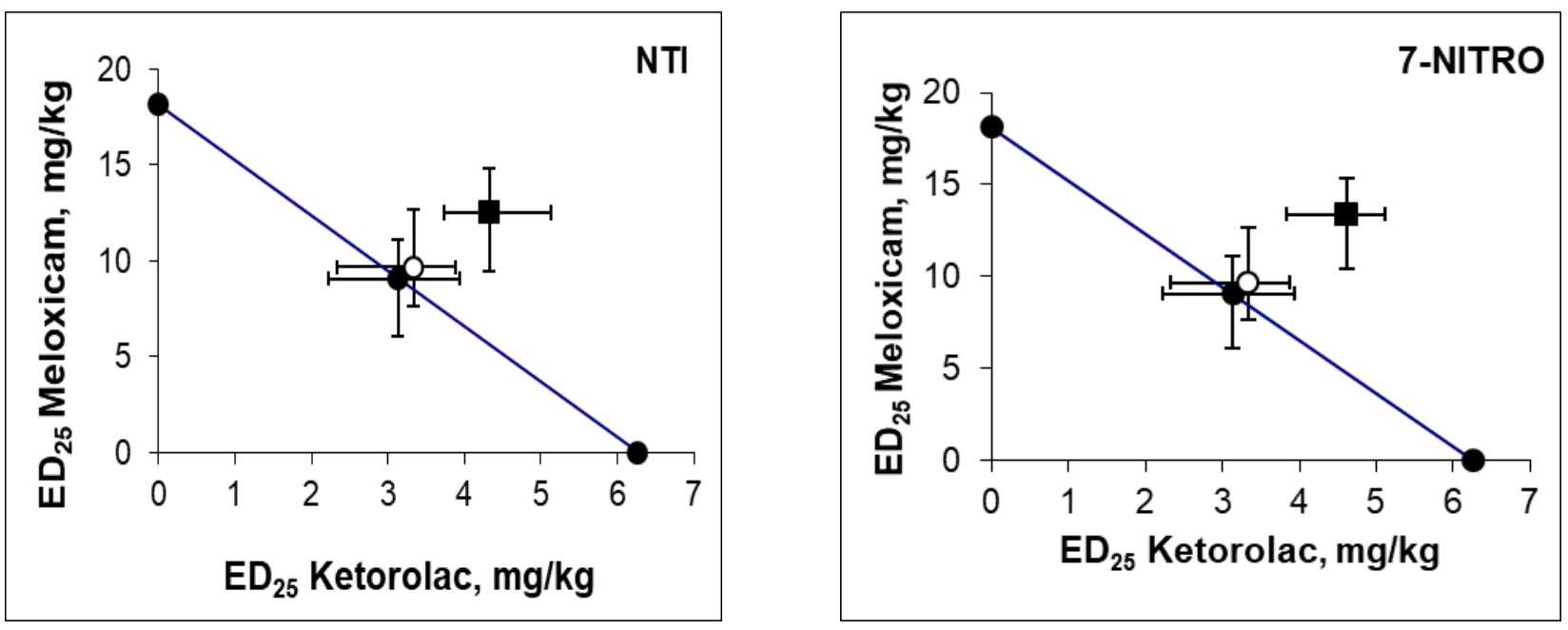

Figure 1 Isobolograms for the administration of ketorolac with meloxicam after pretreatment with naltrindole (NTI) and 7-Nitroindazole (7-Nitro) in the tail flick of mice. (•) theoretical ED25 value with $95 \%$ CL, (O) experimental ED25 value with $95 \%$ CL, ( $)$ post treatment with naltrindole or 7-Nitroindazole with $95 \%$ CL.

The interaction index obtained was $0.967,1,016,1,130$ and 1,163 respectively. Besides, experimental $\mathrm{DE}_{25} \mathrm{Were}_{11.80}$ \pm 1.80 for tropisetron, $12.40 \pm 1.96$ for risperidone, $13.80 \pm 2.75$ for prazosin and $14.00 \pm 2.29$ for yohimbine. See all data in Table 2 and Figure 2. 

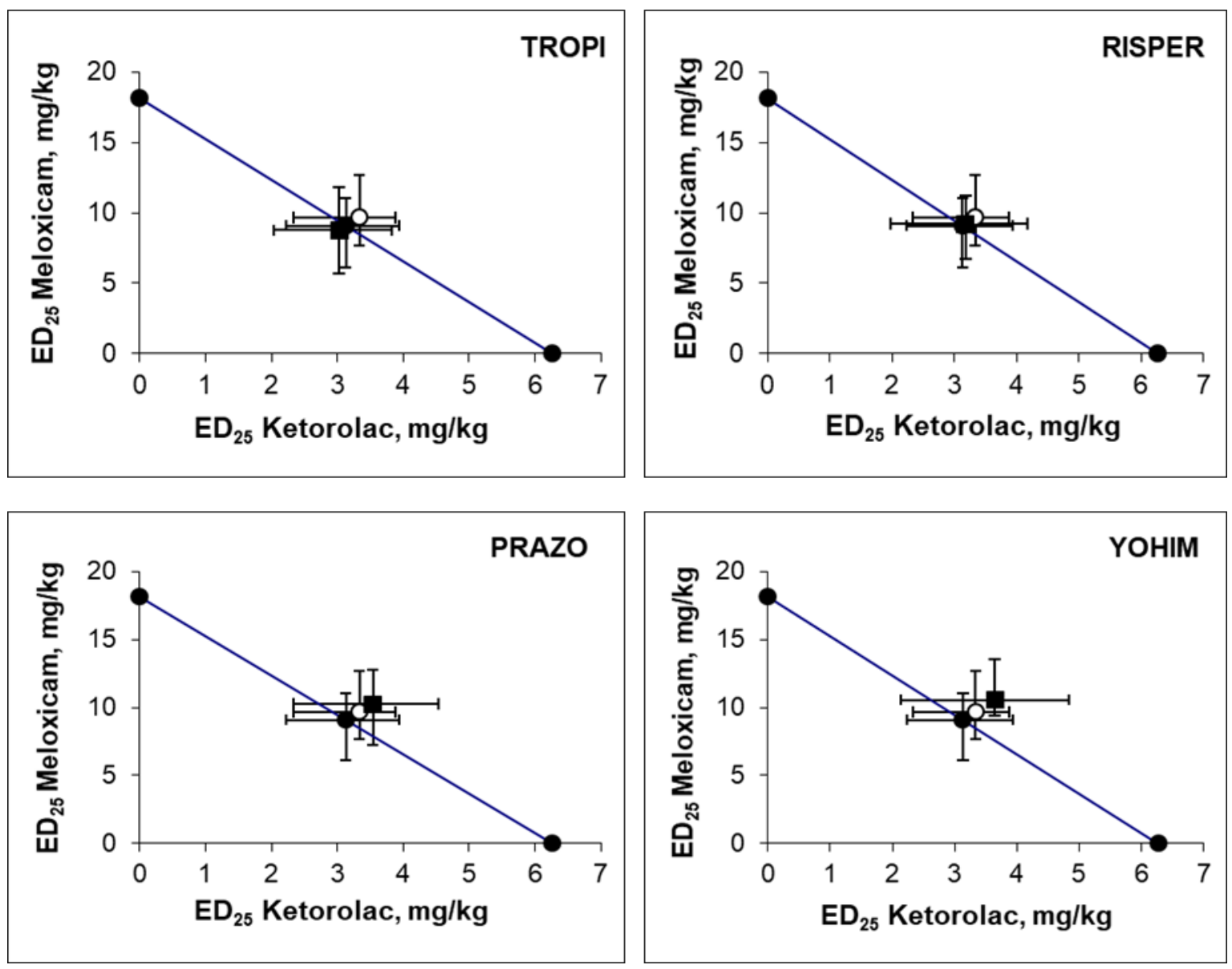

Figure 2 Isobolograms for the administration of ketorolac with meloxicam after pretreatment with tropisetron (TROPI), risperidone (RISPER), prazosin (PRAZO) and yohimbine (YOHIM) in the tail flick of mice. (•) theoretical ED25 value with $95 \%$ CL, (O) experimental ED25 value with $95 \%$ CL, ( $)$ post treatment with antagonist with $95 \%$

CL.

\section{Discussion}

The current work reveals the antinociceptive dose-dependent activity of two NSAIDs: ketorolac and meloxicam, in a phasic pain test, the thermal tail flick of mice. This antinociceptive property of NSAIDs reported is consistent with similar that described in others animal model of pain, such as: hot-plate, acetic acid writhing test and formalin assay [6 -13].

The pharmacological interaction between two drugs can be seen by analyzing the dose response curves, but a more accurate analysis is obtained with the use of isobolographic analysis which measures the effect of the combination using the isobologram. This use a coordinate system and locating a percentage of the effective dose or the maximum effect of each drug on the axes. Depending on whether the effect of the combination is equal to, less than, or greater than the sum of the individual effects, the interaction will be additive, subadditive, or synergistic, respectively.

In the present study carried out in the tail flick test in mice, the interaction obtained between ketorolac (COX-1) and meloxicam (COX-2) was additive, confirmed by an interaction index of 1.065. This finding is difficult to explain since most of the combinations of NSAIDs reported in the scientific literature are synergistic in nature. Pretreatment of mice with tropisetron (5-HT3), or risperidone (D2, 5-HT2, $\alpha 1, \alpha 2$ and H1), or prazosin $(\alpha 1)$ or yohimbine ( $\alpha 2$ ) did not induce modification of the previous additive interaction of ketorolac with meloxicam. This lack of effect produced by the different receptor antagonists is confirmed by the interaction indices between 0.967 and 1,163 respectively. The results obtained demonstrate that the magnitude of the additive interaction between ketorolac and meloxicam was not significantly modified by the antagonists used, which means that the antinociceptive activity of ketorolac and meloxicam is not mediated by serotonergic, dopaminergic, noradrenergic or histaminergic system. 
Nevertheless, if mice are pretreated with naltrindole or 7-Nitroindazole, the additive interaction of ketorolac with meloxicam is significantly modified to a subadditive nature, which is accompanied by an increase in the interaction index to 1.380, in the case of naltrindole and to 1.475 in the case of 7 -Nitroindazole. This modification suggests that ketorolac interaction with meloxicam is dependent on the opioid and nitridergic pathway. The changes induced by naltrindole and by 7-Nitroindazole, were together a decrease in the antinociceptive activity of ketorolac and meloxicam, due to the increase in $\mathrm{ED}_{25}$ values. It is possible to interpret the effects of NTI in the present study, according to the wellknown fact that delta opioid receptors (DOR) mediate antinociceptive effects [14]. In addition, as it has been shown that NTI produce a decrease in analgesic potency [15]. Though, a complex interaction of COX and NO has been observed in multiple systems, among which the binding of COX-2 with the neuronal enzyme nitric oxide synthase (nNOS) stands out [16]. The selective inhibitor of the neuronal enzyme nitric oxide synthase, 7-nitroindazole activates the nuclear factor kappa $\beta$ (NF-kappa $\beta$ ), which leads to an increase in the expression of inflammation [17]. This action of 7-Nitroindazole, is verified in the present study by the change of the interaction of ketorolac with meloxicam from additive to subadditive.

Additionally, it is necessary to add that the results obtained in this study, with the different receptor antagonists used, are compatible with the inhibition of COXs, but require justification by other additional molecular mechanisms, described for NSAIDs, among which we can mention: iNOS inhibition, NF-kappa $\beta$, integrin activation, amandamine (AEA) hydrolysis, cholinergic modulation, depletion of central 5-HT, down regulation of phosphodiesterase tipo IV (PDE $4 D)$, increase of IL-1 $\beta$, L- selectin shedding [18-20].

The current results suggests that the interaction antinociceptive of the combination between ketorolac with meloxicam does not seem to be influenced by adrenergic, dopaminergic, histaminergic or serotonergic mechanisms.

\section{Conclusion}

In conclusion, pretreatment of the mice with naltrindole or con 7-Nitroindazole induced a significant decrease in the antinociceptive power of the combination ketorolac with meloxicam with a significant reduction in $\mathrm{DE}_{25}$. It is suggested that DOR and NO may be involved, in the tail flick model, regulating the antinociceptive bioactivity of these NSAIDs.

\section{Compliance with ethical standards}

\section{Acknowledgments}

None

\section{Disclosure of conflict of interest}

The authors declare that they have no conflict of interest.

\section{Statement of ethical approval}

Experiment were performed in accordance with current Guidelines for the Care of Laboratory Animals and Ethical Guidelines for investigation of experimental pain approved by the Animal Care and Use Committee of the Faculty of Medicine, University of Chile, protocol CBA N $852 / 2018$.

\section{References}

[1] Yam MF, Loh YC, Tan CS, Khadijah AS, Manan A N and Basir R. (2018). General pathways of pain sensation and the major neurotransmitters involved in pain regulation. International Journal of Molecular Sciences, 19, 21632186.

[2] Patrignani P and Patrono C. (2015). Cyclooxygenase inhibitors: From pharmacology to clinical read-outs. Biochimica et Biophysica Acta (BBA) - Molecular and Cell Biology of Lipids, 1851, 422-432.

[3] Brune K and Patrignani P. (2015). New insights into the use of currently available non-steroidal antiinflammatory drugs. Journal of Pain Research, 8, 105-118.

[4] Tallarida RJ. (2001). Drug synergism: its detection and applications. Journal of Pharmacology and Experimental Therapeutics, 298, 865-872. 
[5] Roberts AG and Gibbs ME. (2018). Mechanisms and the clinical relevance of complex drug-drug interactions. Clinical Pharmacology Advance and Application, 10, 123-134.

[6] Miranda HF, Puig MM, Romero MA and Prieto JC. (2009). Effect of tramadol and dexketoprofen on analgesia and gastrointestinal transit in mice. Fundamental and Clinical Pharmacology, 23, 81-88.

[7] Miranda HF, Puig MM, Prieto JC and Pinardi G. (2006). Synergism between paracetamol and nonsteroidal antiinflammatory drugs in experimental acute pain. Pain, 121, 22-28.

[8] González Vargas C, Miranda HF, Sierralta F, Noriega V and Prieto JC. (2019). Pharmacological interaction between NSAIDs with clomipramine and risperidone in mice visceral pain. Drug Development Research, 80, 471- 474 .

[9] González C, Zegpi C, Noriega V, Prieto JC and Miranda HF. (2011) .Synergism between dexketoprofen and meloxicam in an orofacial formalin test was not |modified by opioid antagonists. Pharmacological Reports, $63,433-440$.

[10] Chavarria-Bolaños D, Perez-Urizar J, Grandfils C and Pozos-Guillén A. (2014).Peripheral synergism between tramadol and ibuprofen in the formalin test. Drug Development Reseach, 75, 224-30.

[11] Guevara C, Fernández AC, Cárdenas R and Suarez-Roca H. (2015). Reduction of spinal PGE2 concentrations prevents swim stress-induced thermal hyperalgesia. Neuroscience Letter, 591, 110-114.

[12] Zygmunt M, Chłońrzepa G and Sapa J. (2015). The effect of serotonin 5-HT1 A, 5-HT2 receptor ligands, ketoprofen and their combination in models of induced pain in mice. Acta Poloniae Pharmaceutica, 72, 579585.

[13] Zhou WZ, Zhao TY, Wang ZY, Lu GY, Zhang SZ, Zhang C, Wu N and LI J.(2019). Synergistic antinociception between ZC88, an N-type voltage-dependent calcium channel blocker, and ibuprofen in mouse models of visceral and somatic inflammatory pain. European Journal of Pain, 23, 46 -56.

[14] Jamison RN and Mao J. (2015). Opioids analgesics. Mayo Clinic Proceedings, 90, 957-968.

[15] Willner P, Bergman J and Vanderschuren L. (2020). The behavioural pharmacology of opioids. Biochemical Pharmacology, 31, 109-111.

[16] Alexanian A and Sorokin A. (2017). Cyclooxygenase 2: protein-protein interactions and posttranslational modifications. Physiological Genomics, 49, 667-68.

[17] Glezer I , Munhoz, CD, Kawamoto EM, Marcourakis T, Avellar MC and M C Scavone C. (2003). MK-801 and 7-Ni attenuate the activation of brain NF-kappa B induced by LPS. Neuropharmacology, 45, 1120-1129.

[18] Hamza M and Dionne RA. (2009). Mechanism of non-opioids analgesics beyond cyclooxygenase enzyme inhibition. Current Molecular Pharmacology, 2, 1-14.

[19] Díaz-González F and Sánchez-Madrid F. (2015). NSAIDs: learning new tricks from old drugs. European Journal of Immunology, 45,679-686.

[20] Gunaydin C and Bilge SS. (2018). Effects of nonsteroidal anti-inflammatory drugs at the molecular level. The European Journal of Medicine, 50, 116-121.

\section{How to cite this article}

Noriega V, Sierralta F, Sotomayor-Zárate R, Prieto JC and Miranda HF. (2020). Modulation of synergism COX-1 with COX2 in the tail flick of mice. World Journal of Advanced Research and Reviews, 6(3), 222-228. 